\title{
BMJ Open MinT-trial: Mindfulness versus cognitive behavioural therapy in Tinnitus patients: protocol for a randomised controlled, non- inferiority trial
}

\author{
Maaike Maartje Rademaker (10), ${ }^{1,2}$ Inge Stegeman, ${ }^{1,2}$ Arno Lieftink, \\ Metten Somers, ${ }^{3}$ Robert Stokroos, ${ }^{1,2}$ Adriana L Smit ${ }^{1,2}$
}

To cite: Rademaker MM, Stegeman I, Lieftink A, et al. MinT-trial: Mindfulness versus cognitive behavioural therapy in Tinnitus patients: protocol for a randomised controlled, non-inferiority trial. BMJ Open 2020;10:e033210. doi:10.1136/ bmjopen-2019-033210

- Prepublication history and additional material for this paper are available online. To view these files, please visit the journal online (http://dx.doi. org/10.1136/bmjopen-2019033210).

Received 25 July 2019

Revised 20 January 2020

Accepted 29 January 2020

A) Check for updates

(C) Author(s) (or their employer(s)) 2020. Re-use permitted under CC BY-NC. No commercial re-use. See rights and permissions. Published by BMJ.

For numbered affiliations see end of article.

\section{Correspondence to} Maaike Maartje Rademaker; m.m.rademaker-3@umcutrecht. $\mathrm{nl}$

\section{ABSTRACT}

Introduction Chronic subjective tinnitus is a condition that affects $5.1 \%$ to $42.7 \%$ of the population, depending on the definition and studied population. Evidence-based treatment options are limited. Cognitive Behavioural Therapy (CBT) has been proven effective to improve quality of life and to diminish tinnitus distress. Positive short-term effects of mindfulness-based interventions on tinnitus distress have been reported; however, the longer term effects remain to be studied.

Methods and analysis We designed a monocentre randomised controlled, non-inferiority trial to compare the effectiveness of mindfulness-based cognitive therapy (MBCT) and CBT in chronic tinnitus patients. Fifty-four patients ( $\geq 32$ on the Tinnitus Functional Index (TFI), suffering from tinnitus for at least 6 months) will be included in the trial and randomised into one of two intervention groups. One group will receive MBCT, the other group will receive CBT. Our primary objective is to determine whether MBCT is non-inferior to (as good as) CBT on tinnitus distress (TFI) in chronic tinnitus patients at 12 months follow-up after end of therapy. Non-inferiority will be declared if the mean decrease in TFI score for MBCT is no worse than the mean decrease in TFI score in CBT, with statistical variability, with a margin of 13 points. Most secondary objectives (tinnitus severity of problem, tinnitus intrusiveness, quality of life, anxiety, depression, symptoms of psychopathology, perceived tinnitus complaints, coping style (mostly validated questionnaires)) are expected to show non-inferiority to MBCT compared with CBT. We expect a significant difference between MBCT and CBT for mindfulness awareness.

Ethics and dissemination This research protocol was approved by the Institutional Review Board of the UMC Utrecht (NL67838.041.18, V.4, April 2019). The trial results will be made accessible to the public in a peer-review journal.

Trial registration number NL7745.

\section{BACKGROUND}

Tinnitus is the perception of sound in the absence of acoustic stimulation and is often experienced as a ringing or buzzing sound. ${ }^{1}$
Strengths and limitations of this study

- This study allows for a comparison between mindfulness-based cognitive therapy (MBCT) and cognitive behavioural therapy (CBT, standard care).

- This is the first high-quality non-inferiority trial to evaluate long-term effects of MBCT in chronic tinnitus patients.

- Multiple outcome measures will be assessed with validated questionnaires, such as but not limited to tinnitus distress, anxiety and depression, coping style and quality of life.

Differences in the amount of therapy hours, and composition of the group in both treatments will limit generalisability and might influence the outcome.

- Though, CBT for tinnitus and MBCT will be compared, both interventions might entail elements of the other intervention.

Over 70 million people in Europe suffer from some form of tinnitus, which can be chronic and disabling for affected individuals. ${ }^{2}$ The perceived distress is caused by tinnitus characteristics such as loudness and severity and by additional complaints such as difficulties in concentration, sleeping problems and feelings of anxiety. ${ }^{3-5}$ Quality of life decreases with increasing tinnitus complaints, and patients with severe tinnitus disturbance can experience severe limitations in their dayto-day life. ${ }^{16}$

Treatment modalities for tinnitus can be divided in pharmacological and nonpharmacological therapies. ${ }^{7}$ So far, no curative treatment has been identified and evidence-based treatment options for tinnitus are limited, which might be related to its heterogeneity, perception of tinnitus and levels of distress. ${ }^{8}$ 
Cognitive behavioural therapy (CBT) has been proven effective in tinnitus patients to improve quality of life and diminish tinnitus distress. ${ }^{9}{ }^{10}$ In CBT, a structured psychological intervention, patients are taught cognitive and behavioural skills to influence (responsive) thoughts and behaviour. It is based on the idea that people's experiences influence their concepts. ${ }^{9}$ Treatment therefore focusses on unhelpful thoughts and behaviour. CBT has developed over the years in regard to techniques and delivery methods. These are most commonly referred to as three 'waves'. ${ }^{11}$ While operant learning and conditioning methods are highlighted in 'first wave' methods, in the 'second wave', the attention is more aimed at handling information. ${ }^{12}$ More recent developments in CBT, also referred to as 'third-wave', stress acceptance and mindfulness. ${ }^{13}$

Mindfulness meditation originates from Buddhist philosophy and psychology and is defined as 'the process of openly attending, with awareness, to one's present moment experience' ${ }^{14}$ (p493). It aims to achieve a non-judgemental approach to thought, emotions and sounds. ${ }^{14}$ Dr Kabat-Zinn first introduced elements of mindfulness meditation to western medicine in a systematically delivered group intervention called mindfulnessbased stress reduction to treat chronic pain. ${ }^{15}$ Over time, different types of psychological interventions that use elements of mindfulness meditation have been developed; these are often referred to as a mindfulness-based intervention (MBI) ${ }^{14}$ A common structured group-based intervention is mindfulness-based cognitive therapy (MBCT). MBCT was originally developed for relapsing depression. ${ }^{15-17}$ In MBCT, elements of CBT techniques are combined with mindfulness meditation. ${ }^{14}{ }^{16}$ MBIs are known to have beneficial effects in treating relapsing depression, anxiety, stress and chronic pain. ${ }^{18-21}$

The question why CBT, including 'third wave' methods, may reduce tinnitus distress can be explained by several, partly overlapping theories that explain mechanisms of tinnitus tolerance. It is thought that habituation and cognitive approaches interfere with defective behavioural and emotional reactions that are thought to maintain tinnitus distress. ${ }^{22-24}$ Avoidance, considered a fear condition reaction, is correlated with tinnitus severity and distress. ${ }^{23}{ }^{25}$ Mindfulness meditation is believed to have a beneficial effect on tinnitus distress due to its focus on acceptance, the opposite of avoidance. ${ }^{2326}$ Also, cognitive and metacognitive awareness are thought to be enhanced by mindfulness mediation. This helps to diminish the emotional damages of negative cognitions that are associated with tinnitus severity. ${ }^{22} 27-29$

Several research groups have investigated the effectiveness of MBIs, in either randomised controlled trials, cohort studies or a comparative controlled trial. ${ }^{27} 28$ 30-35 The majority of observational studies show a decrease in tinnitus distress after MBI. ${ }^{27} 28$ 30-34 36 Two out of three published randomised controlled trials show a statistically superior decrease of tinnitus distress compared with a control group of relaxation therapy directly

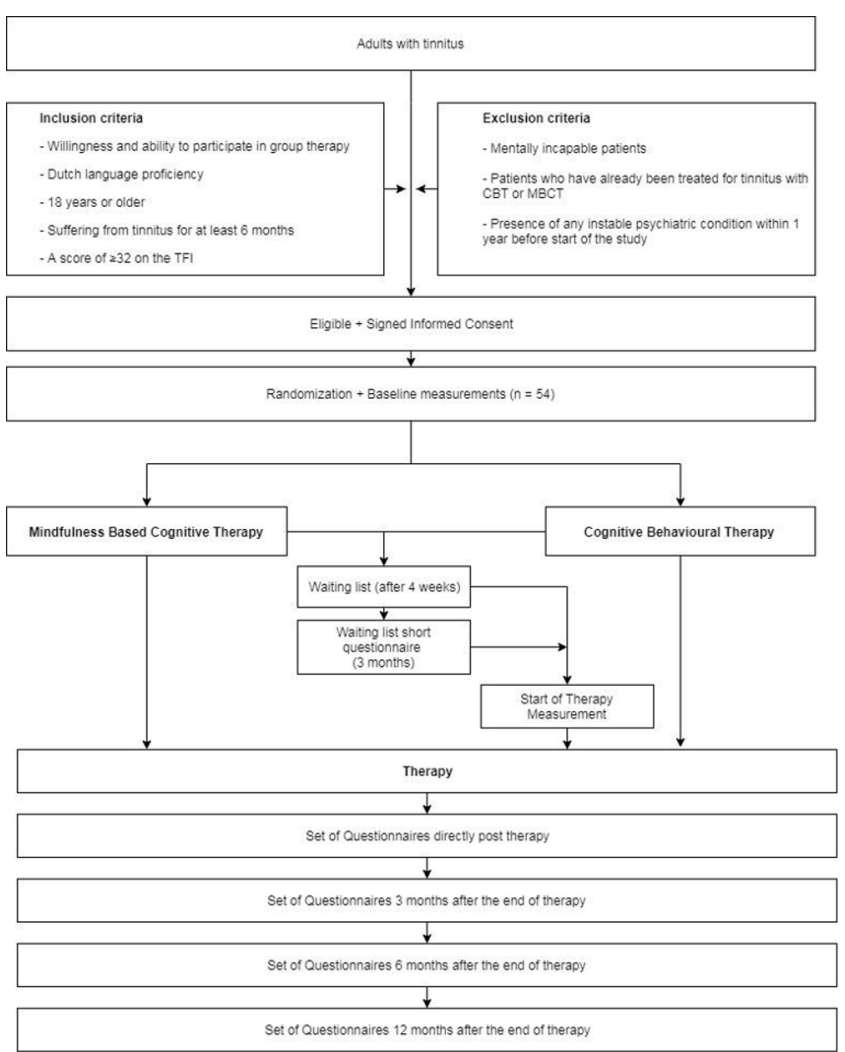

Figure 1 Study flowchart. CBT, cognitive behavioural therapy; MBCT; mindfullness-based cognitive thearpy; TFI, Tinnitus Functional Index.

after treatment. ${ }^{28} 3031$ One study demonstrated results at 6 months after therapy in which this positive result remained significant. ${ }^{28}$ In a letter to the editor, Gans $e t$ al additionally described a sustained positive effect of their MBI in terms of tinnitus distress relief 1 year after treatment in seven patients. ${ }^{37}$

Based on the short-term outcomes, mindfulness is effective in reducing tinnitus distress, in this study the longer term outcomes will be investigated (12 months). In this randomised study, the effect on tinnitus distress by MBCT versus CBT in patients with chronic tinnitus will be compared. We hypothesise a non-inferiority of tinnitus distress in MBCT compared with CBT.

\section{METHODS AND ANALYSIS Study objectives}

The primary objective of this study is to compare the effect of MBCT and CBT on tinnitus distress, measured with the Tinnitus Functional Index (TFI) at 12 months after end of therapy. ${ }^{38}$ Secondary objectives are to compare the effect of MBCT and CBT on tinnitus severity, quality of life, anxiety, depression, other symptoms of psychopathology, mindfulness awareness, the subjective effect of therapy and coping style.

\section{Study design and setting}

In this non-inferiority RCT, patients will be randomised into two groups (figure 1). This is a monocentre study 
performed in a tertiary referral clinic (university hospital) in the Netherlands (University Medical Center Utrecht). The protocol is reported according to the Standard Protocol Items: Recommendations for Interventional Trials statement. ${ }^{39}$

We will declare non-inferiority if the mean decrease in TFI score for MBCT is no worse than the mean decrease in TFI score in CBT, with statistical variability, with a margin of 13 points. Almost all secondary objectives (tinnitus severity (severity of problem and intrusiveness), quality of life, anxiety and depression, symptoms of psychopathology, perceived tinnitus complaints, coping style (all validated questionnaires)) are expected to show noninferiority of MBCT compared with CBT. We expect a significant difference between MBCT and CBT for mindfulness awareness.

\section{Study population}

The study population consists of patients who are referred to, diagnosed and/or counselled by the Tinnitus Care group of the outpatient department of Otorhinolaryngology of the University Medical Center Utrecht as a secondary and tertiary referral centre for tinnitus. Patients will be asked for permission to be contacted by the research team. The following criteria must be met to be eligible for inclusion. All elements of eligibility, except for the TFI score, will be judged by taking medical history.

\section{Inclusion criteria}

- Willingness and ability to participate in group therapy.

- Dutch language proficiency.

- 18 years or older.

- Suffering from chronic tinnitus for at least 6 months.

- A score of $\geq 32$ on the TFI.

\section{Exclusion criteria}

A potential subject who meets any of the following criteria will be excluded from participation in this study.

- Mentally incapable patients.

- Patients who have already been treated for tinnitus with CBT or MBCT.

- Presence of any instable psychiatric condition within 1 year before start of the study.

If eligibility is judged and approved for inclusion by the research team, patients will be sent the patient information letter and an informed consent (IC) form (online supplementary material 1 ). Patients will be given 2 weeks to consider participation. If participants decide not to take part, they will continue within the regular care of the tinnitus line of care in the UMC Utrecht. The IC form will be signed by the patient and a member of the research team.

\section{Randomisation and interventions}

After inclusion and baseline assessment, patients will be randomly allocated into one of two intervention groups (MBCT or CBT). Block randomisation will be electronically performed by the study management system Castor Electronic Data Capture (EDC). ${ }^{40}$ Both groups carry the same weight (1:1). Block groups will be 2 , 4 . Investigators will be blinded to the randomisation sequence. Blinding of participant and care providers is not possible due to the nature of the intervention.

Intervention group A will receive 8 weekly sessions of MBCT; each session will last for two and a half hours (total 20 hours). All trainings will be supervised by a certified, experienced mindfulness trainer (experienced with psychological and psychiatric diseases). The MBCT is standard care in the psychiatry department of the UMC Utrecht. The study participants will join this group. The MBCT has not been adapted for tinnitus patients. Treatment group B will receive 5 weekly CBT sessions (solely tinnitus patients) and one refreshment session 2 to 3 months later, as a standard of care treatment. The CBT was specifically designed for tinnitus patients. The therapy will be supervised by a social worker and a psychologist, who both have several years of experience in CBT training for tinnitus patients. The CBT sessions will last approximately 2 hours (total 12 hours). The exact content of the intervention and control group can be found in table 1 .

Due to potentially limited capacity in both treatment groups, participants might have to wait a maximum of 6 months before the start of both treatments. A waiting list control group will be applicable for both treatment groups. The waiting list can last up to 6 months. Participants who have to wait for more than 4 weeks for therapy from the moment of randomisation will be on the waiting list.

Participants are not allowed to start another tinnitus treatment during the course of the study.

\section{Sample size}

To achieve a power of $80 \%$, with an alpha of $5 \%$, a noninferiority test of two means (T-test) was performed. This resulted in a sample size of 25 patients per group. To correct for possible drop outs, we will include 54 patients, with about 27 patients per arm. The low dropout rate was based on the motivated patient group. For this noninferiority test, we used the primary end point of the TFI outcome at 12 months. We determined the equivalence margin to be 13 points. This is based on the minimally clinically important difference (MCID) of the TFI. ${ }^{38}$ The acceptable SD was set at 18 points, based on the results of a previous RCT on mindfulness in tinnitus patients. ${ }^{28}$

\section{Outcomes}

At intake baseline data will be collected. If necessary, patients will undergo audiometric tests. This will only happen when the audiometric tests were older than 6 months at the date of inclusion. Audiometric tests will consist of pure tone and speech audiometry, tinnitus loudness match, tinnitus pitch match and tinnitus maskability.

At inclusion, demographic data will be extracted from the electronic patient database: gender, age, highest level of education, marital status, working situation, number of 
Table 1 Detailed content of both intervention groups

\section{Content of both intervention groups}

\begin{tabular}{ll}
\hline MBCT & CBT \\
-8 weekly courses & 5 weekly course, 1 refreshment \\
& session after 2-3 months \\
Certified, experienced & Experienced psychologist and \\
mindfulness trainers & experienced social worker
\end{tabular}

\section{Session 1}

$\begin{array}{ll}\text { Life on autopilot } & \text { Information and acquaintance } \\ \text { Introduction } & \text { Introducing supervisors } \\ \text { Acquaintance } & \text { Organisational and practical } \\ \text { Practical information } & \text { information } \\ \text { Eat meditation and } & \text { Group rules } \\ \text { evaluation } & \text { Inventory and comparison } \\ \text { Theme } & \text { Objectives and design of the } \\ \text { Pause } & \text { training } \\ \text { Bodyscan and evaluation } & \text { Exercise: distraction from } \\ \text { Explanation of course } & \text { tinnitus } \\ \text { book and practice } & \\ \text { Session 2 } & \\ \text { Dealing with obstacles } & \text { Tinnitus and distraction } \\ \text { Bodyscan and evaluation } & \text { Discuss content and exercise } \\ \text { Pause } & \text { session } \\ \text { Theme } & \text { Tinnitus and distraction } \\ \text { Evaluation home } & \text { Decreasing tinnitus' influence } \\ \text { exercise, discuss next } & \text { Ways of distraction } \\ \text { week's home exercise } & \text { Choosing a new distraction } \\ \text { Closing } & \text { Exercise: a new way of } \\ & \text { distraction }\end{array}$

\section{Session 3}

$\begin{array}{ll}\text { Exploring your boundaries } & \text { Tinnitus and attention } \\ \text { Opening meditation } & \text { Discuss content and exercise } \\ \text { Explanation breathing } & \text { session } 2 \\ \text { space and evaluation } & \text { Tinnitus and attention } \\ \text { Theme } & \text { Practice shifting of focus } \\ \text { Evaluation home } & \text { Exercise breathing/relaxation } \\ \text { exercise, discuss next } & \text { Techniques decreasing focus } \\ \text { week's home exercise } & \text { Exercise: apply } \\ \text { Meditation lying down } & \text { focus-technique } \\ \text { Closing } & \\ \text { Session 4 } & \\ \text { Stress } & \text { Tinnitus and meaning } \\ \text { Auditory and visual } & \text { Exercise breathing/relaxation } \\ \text { meditational exercise } & \text { Discuss content and exercise } \\ \text { Standing meditation and } & \text { session 3 } \\ \text { evaluation } & \text { Exercise progressive muscle } \\ \text { Theme } & \text { relaxation } \\ \text { Evaluation home } & \text { Tinnitus and thoughts, formula: } \\ \text { exercise, discuss next } & \text { G+G = G+G } \\ \text { week's home exercise } & \text { Possibilities of thought } \\ \text { Sedentary meditation } & \text { alteration } \\ \text { and evaluation } & \text { Exercise: administering though } \\ & \text { alteration }\end{array}$

\section{Session 5}

Conscious handling of Tinnitus and relaxation
challenges

Continued

\section{Table 1 Continued}

\section{Content of both intervention groups}

- Opening mediation

- Sedentary meditation and evaluation

- Evaluation home exercise

- Theme

- Discuss next week's home exercise

- Walking meditation

- Closing

\section{Session 6}

$\begin{array}{ll}\text { Thought are not facts } & \text { Refreshment } \\ \text { Meditation, extra focus } & \text { Exchanging experiences } \\ \text { on sedentary meditation } & \text { Refreshing course content } \\ \text { and evaluation } & \text { Evaluation and closing } \\ \text { Evaluation home } & \\ \text { exercise } \\ \text { Theme } \\ \text { Discuss next week's } \\ \text { home exercise } \\ \text { Walking meditation and } \\ \text { physical exercise }\end{array}$

Session 7

\section{Taking care of yourself}

- Opening meditation

- Sedentary meditation and evaluation

- Evaluation home exercise

- Theme

- Discuss next week's home exercise

- Walking meditation and physical exercise

- Closing

Session 8
Opening meditation
Bodyscan
Evaluation bodyscan
Evaluation home
exercise
- Tips on integrating
mindfulness in your life
- Evaluation training
Closing

CBT, cognitive behavioural therapy; MBCT, mindfullness-based cognitive therapy.

people in the household, average income of household, native language and postal codes.

Outcome measures will be assessed by questionnaires. These questionnaires will be sent by e-mail to the study participants through the data management programme Castor EDC..$^{40}$ If participants do not want to receive the set of questionnaires electronically, they will receive them by postal services. Patients will receive the different questionnaires at five moments in time; at baseline, end of 
Table 2 Timing of different questionnaires

\begin{tabular}{|c|c|c|c|c|c|c|}
\hline Questionnaire & Baseline/s.o.t.* & Waiting list ( 3 months $\dagger$ ) & FU e.o.t.‡ & FU 3 months & $\begin{array}{l}\text { FU } 6 \\
\text { months }\end{array}$ & $\begin{array}{l}\text { FU } 12 \\
\text { months }\end{array}$ \\
\hline TSCHQ & $x$ & & & & & \\
\hline Tinnitus VAS scales & $x$ & $x$ & $x$ & $x$ & $x$ & $x$ \\
\hline HADS & $x$ & & & $x$ & & $x$ \\
\hline CGI-CHANGE & & & $x$ & $x$ & $x$ & $x$ \\
\hline MAAS & $x$ & & & $x$ & & $x$ \\
\hline SCL-90-R & $x$ & & & & & $x$ \\
\hline General Questions§ & $X$ & $x$ & $x$ & $x$ & $X$ & $X$ \\
\hline
\end{tabular}

${ }^{*}$ s.o.t.: start of treatment (applicable for participants if therapy starts more than 4 weeks after baseline measurement).

†Data on the waiting list questionnaire will be valid for 4 weeks. If participants start treatment within 4 weeks after this short questionnaire, they will not be asked to repeat these questionnaires (TFI, tinnitus VAS scales, questions about medication, comorbidities, etc) before the start of treatment.

‡e.o.t.: end of treatment.

§General questions include questions on medication, comorbidities, additional tinnitus therapies and adverse events.

CGI-CHANGE, Clinical Global Impression; CISS, Coping Inventory for Stressful Situation; FU, follow-up; HADS, Hospital Anxiety and Depression Scale; MAAS, Mindful Attention Awareness Scale; SCL, symptom checklist-90-R; TFI, Tinnitus Functional Index; TSCHQ, Tinnitus Sample Case History Questionnaire; VAS, visual analogue scale; WHOQOL-BREF, WHO Quality of Life (Questionnaire)-BREF.

treatment (MBCT after session eight, CBT after session six) and after 3, 6 and 12 months follow-up after end of treatment (table 2).

If participants are on the waiting list ( $>4$ weeks before start therapy after study inclusion), they will be required to fill out a set of questionnaires (identical to the baseline set of questionnaires) before the start of treatment. If participants are on the waiting list for 3 months, they will be sent a short questionnaire during the waiting list period. This questionnaire will be valid for 4 weeks. If participants start treatment within 4 weeks after this short questionnaire, they will not be asked to repeat these questionnaires (TFI, tinnitus visual analogue scale (VAS) scales, questions about medication, comorbidities, etc) before the start of treatment (table 2).

\section{Explanation of different questionnaires}

- TFI, consisting out of 25 questions to measure the impact of tinnitus on daily life on 11-point Likert scales. The TFI was translated to Dutch and validated in 2014, with a reported Cronbach $\alpha=0.91$. The TFI consists of eight subscales to assess intrusiveness, sense of control, cognition, sleep, hearing (auditory), relaxation, quality of life and emotions (emotional). The score of the TFI ranges from 0 to 100 , with an MCID of 13. The scores can be stratified into five levels: $0-17=$ nota problem, $18-31=$ small problem, $32-53=$ moderate problem, 54-72=big problem, 73-100=verybig problem. ${ }^{38} 41$

- Tinnitus Sample Case History Questionnaire; 35 questions assessing the tinnitus history.

- Tinnitus VAS scales; two questions regarding the severity of tinnitus patients might experience scored on a VAS scale (severity of the tinnitus (one no problem to five very large problem) and intrusiveness (0 not intrusive at all-10 extremely intrusive).

- WHO Quality of Life (Questionnaire)-BREF (WHOQOL-BREF); 26 questions to measure participants' quality of life on a five-point Likert scale. The WHOQOL-BREF was translated to and validated in Dutch in 2005. The WHOQOL-BREF measures four subdomains: physical health (Cronbach $\alpha=0.80$ ), psychological health (Cronbach $\alpha=0.74$ ), social relationships (Cronbach $\alpha=0.66$ ) and environment (Cronbach $\alpha=0.73){ }^{42}$

- Hospital Anxiety and Depression Scale (HADS); 14 questions to assess anxiety and depression on four point scales. It consists of two scales of seven items, one for anxiety and the other for depression. Cronbach $\alpha$ ranges between 0.71 and 0.90 on both subscales and the total scale. Total scores range from 0 to 21 per scale. The HADS was translated to and validated in Dutch in $1997 .{ }^{43}$

- Mindful Attention Awareness Scale (MAAS); 15 questions to assess mindfulness awareness on a six-point Likert scale ( $1=$ almost always to $6=$ almost never). The MAAS was translated to and validated in Dutch in 2008, Cronbach $\alpha$ ranging from 0.80 to 0.85 . A mean of the sum of scores is calculated to score the MAAS. ${ }^{1-6}$ Higher scores indicate more mindfulness. ${ }^{44}$

- Questions to assess whether participants started to use concomitant medication suffer from comorbidities, adverse events (AE) or started another treatment for their tinnitus during the course of the study. 
- Coping Inventory for Stressful Situation; 48 questions to assess patient's coping style on a five-point Likert scale ( $1=$ not at all, $5=$ very much). There are three subscales: task-oriented, emotion-oriented and avoidance-oriented. Avoidance-oriented can be divided into two other subscales: distraction and social diversion. Cronbach $\alpha$ is above 0.80 for taskoriented, emotion-oriented and avoidance-oriented coping, and higher than 0.70 for distraction and social diversion. ${ }^{45}$

- Clinical Global Impression scale; one question to assess subjective improvement of tinnitus after therapy, scored on a scale of 1 (very much improved) to 7 (very much worse). ${ }^{46}$

- SCL-90-R; 90 questions to assess symptoms of psychopathology on a five-point scale for not at all $=0$ to $4=$ extremely. Translated and validated in Dutch in 2004, Cronbach $\alpha$ range between 0.76 and 0.97 over the subscales in the general population. There are nine subscales: somatisation, obsessive-compulsive, interpersonal sensitivity, depression, anxiety, hostility, phobic anxiety, paranoid ideation and psychoticism. ${ }^{47}$

\section{Statistical analysis}

Baseline characteristics will be presented in means or medians, depending on the normality of the data. Categorical data will be presented quantitatively. For the longitudinal analysis, a mixed-model analysis will be performed. Non-inferiority will be established when both approaches support non-inferiority. A margin of 13 point on the TFI is defined as non-inferiority. We expect limited missing data, due to the motivated nature of the patient population. Potentially missing variables will be handled with multiple imputation, a complete case analysis will be performed as a sensitivity analysis. A p value of $<0.05$ is considered statistically significant. Intention to treat and per protocol analysis will be performed. No interim analyses will be performed.

Analyses of between-group differences of the secondary outcomes measures will be performed with a mixedmodel analysis.

\section{ETHICS AND DISSEMINATION}

This study will be conducted according to the principles of the Declaration of Helsinki (64th WMA General Assembly, Fortaleza, Brazil, October 2013) and in accordance with the Medical Research Involving Human Subjects Act (WMO), the 'gedragscode gezondheidsonderzoek' and 'code goed gebruik'.

Protocol amendments will be notified to the local Medical Research Ethics Committee (MREC). The trial results will be made accessible to the public in a peerreview journal.

All data will be handled confidentially. The data will be analysed anonymously by using a unique patient identification number. Data will be collected through Castor EDC and data will be pseudonymised. The investigator will safeguard the key to the code. Patient data will be stored on a password-protected computer in a lockable room. IC forms will be kept in a locked cabin. If a patient does not wish to fill out the questionnaires electronically, they will receive them by postal services. Data will be administered in Castor by an investigator to the correct patient. The final trial data set will be safeguarded and available to the principal investigator and approved members of the research team.

Trial quality will be independently monitored by a local monitor (UMC Utrecht) once a year. The local monitor will check $10 \%$ of signed ICs, inclusion and exclusion criteria, source data and serious adverse events (SAE). From the first three participants, the inclusion and exclusion criteria will also be checked. The speed of inclusion, drop-out rate and the presence and completeness of the monocentre study file will be monitored. $\mathrm{AE}$ will be recorded; SAE will be reported to the local Institutional Review Board.

\section{Trial status}

The trial is currently in recruitment phase. The first patient was recruited on 29 October 2019. Nine of 54 patients were included in the study on 17 January 2020.

\section{Author affiliations}

${ }^{1}$ Department of Otorhinolaryngology, Head and Neck Surgery, University Medical Center Utrecht, Utrecht, The Netherlands

${ }^{2}$ Department of Otorhinolaryngology, University Medical Center Utrecht Brain Center, Utrecht University, Utrecht, The Netherlands

${ }^{3}$ Department of Psychiatry, University Medical Center Utrecht, Utrecht, The Netherlands

Contributors MMR developed the protocol, drafted the manuscript, revised the manuscript and approved the final version. MS, RS, AL, IS and ALS developed the protocol, revised the manuscript and approved the final version.

Funding Dutch Rehabilitation Fund ("Revalidatie fonds")

Competing interests None declared.

Patient consent for publication Not required.

Ethics approval This research protocol was approved by the Institutional Review Board (IRB) of the UMC Utrecht (NL67838.041.18, V.4, April 2019).

Provenance and peer review Not commissioned; externally peer reviewed.

Open access This is an open access article distributed in accordance with the Creative Commons Attribution Non Commercial (CC BY-NC 4.0) license, which permits others to distribute, remix, adapt, build upon this work non-commercially, and license their derivative works on different terms, provided the original work is properly cited, appropriate credit is given, any changes made indicated, and the use is non-commercial. See: http://creativecommons.org/licenses/by-nc/4.0/.

ORCID iD

Maaike Maartje Rademaker http://orcid.org/0000-0002-4904-1394

\section{REFERENCES}

1 Langguth B, Kreuzer PM, Kleinjung T, et al. Tinnitus: causes and clinical management. Lancet Neurol 2013;12:920-30.

2 Fackrell K, Smith H, Colley V, et al. Core Outcome Domains for early phase clinical trials of sound-, psychology-, and pharmacologybased interventions to manage chronic subjective tinnitus in adults: the COMIT'ID study protocol for using a Delphi process and face-toface meetings to establish consensus. Trials 2017;18:1-11.

3 Hoekstra CEL, Wesdorp FM, van Zanten GA. Socio-Demographic, health, and tinnitus related variables affecting tinnitus severity. Ear Hear 2014;35:544-54. 
4 Stouffer JL, Tyler RS. Characterization of tinnitus by tinnitus patients. J Speech Hear Disord 1990;55:439-53.

5 Tyler RS, Baker LJ. Difficulties experienced by tinnitus sufferers. $J$ Speech Hear Disord 1983;48:150-4.

6 Zeman F, Koller M, Schecklmann M, et al. Tinnitus assessment by means of standardized self-report questionnaires: psychometric properties of the tinnitus questionnaire (TQ), the tinnitus handicap inventory (THI), and their short versions in an international and multilingual sample. Health Qual Life Outcomes 2012;10:128.

7 Fernández MM, Maldonado Fernández M, et al. Cochrane database of systematic reviews interventions for tinnitus in adults: an overview of systematic reviews (protocol) I interventions for tinni. Cochrane Database Syst Rev Art 2015;7.

8 Cederroth CR, Gallus S, Hall DA, et al. Editorial: towards an understanding of tinnitus heterogeneity. Front Aging Neurosci 2019;11:1-7

9 Martinez-Devesa P, Perera R, Theodoulou M, et al. Cognitive behavioural therapy for tinnitus. Cochrane Database Syst Rev 2010:CD005233.

10 Cima RFF, Maes IH, Joore MA, et al. Specialised treatment based on cognitive behaviour therapy versus usual care for tinnitus: a randomised controlled trial. The Lancet 2012;379:1951-9.

11 Landry EC, Sandoval XCR, Simeone CN, et al. Systematic review and network meta-analysis of cognitive and/or behavioral therapies (CBT) for tinnitus. Otol Neurotol 2020;41:153-66.

12 Carvalho S, Martins CP, Almeida HS, et al. The evolution of cognitive behavioural therapy - The third generation and its effectiveness. European Psychiatry 2017;41:S773-4.

13 Hayes SC, Hofmann SG. The third wave of cognitive behavioral therapy and the rise of process-based care. World Psychiatry 2017:16:245-6.

14 Creswell JD, Interventions M. Mindfulness interventions. Annu Rev Psychol 2017;68:491-516.

15 Kabat-Zinn J. An outpatient program in behavioral medicine for chronic pain patients based on the practice of mindfulness meditation: theoretical considerations and preliminary results. Gen Hosp Psychiatry 1982;4:33-47.

16 Segal ZV, Williams JMG, Teasdale JD. Mindfulness based cognitive therapy for depression: a new approach to preventing relapse. New York: Guildford Press, 2002

17 Kabat-Zinn J, Massion AO, Kristeller J, et al. Effectiveness of a meditation-based stress reduction program in the treatment of anxiety disorders. Am J Psychiatry 1992;149:936-43.

18 Bohlmeijer E, Prenger R, Taal E, et al. The effects of mindfulnessbased stress reduction therapy on mental health of adults with a chronic medical disease: a meta-analysis. $J$ Psychosom Res 2010;68:539-44.

19 Hofmann SG, Sawyer AT, Witt AA, et al. The effect of mindfulnessbased therapy on anxiety and depression: a meta-analytic review. $J$ Consult Clin Psychol 2010;78:169-83.

20 Morone NE, Greco CM, Weiner DK. Mindfulness meditation for the treatment of chronic low back pain in older adults: a randomized controlled pilot study. Pain 2008:134:310-9.

21 Wong SY-S, Chan FW-K, Wong RL-P, et al. Comparing the effectiveness of mindfulness-based stress reduction and multidisciplinary intervention programs for chronic pain: a randomized comparative trial. Clin J Pain 2011;27:724-34.

22 McKenna L, Handscomb L, Hoare DJ, et al. A scientific cognitivebehavioral model of tinnitus: novel conceptualizations of tinnitus distress. Front Neurol 2014;5:1-15.

23 Cima RFF. Bothersome tinnitus : Cognitive behavioral perspectives. HNO 2018;66:369-74.

24 Jastreboff PJ. Phantom auditory perception (tinnitus): mechanisms of generation and perception. Neurosci Res 1990;8:221-54.

25 Hesser H, Andersson G. The role of anxiety sensitivity and behavioral avoidance in tinnitus disability. Int J Audiol 2009;48:295-9.
26 Hesser H, Bånkestad E, Andersson G. Acceptance of tinnitus as an independent correlate of tinnitus severity. Ear Hear 2015;36:e176-82.

27 McKenna L, Marks EM, Vogt F. Mindfulness-Based cognitive therapy for chronic tinnitus: evaluation of benefits in a large sample of patients attending a tinnitus Clinicl link. Ear Hear 2017:1-8.

28 McKenna L, Marks EM, Hallsworth CA, et al. Mindfulness-Based cognitive therapy as a treatment for chronic tinnitus: a randomized controlled trial. Psychother Psychosom 2017;86:351-61.

29 Cima RFF, Crombez G, Vlaeyen JWS. Catastrophizing and fear of tinnitus predict quality of life in patients with chronic tinnitus. Ear Hear 2011;32:634-41.

30 Arif M, Sadlier M, Rajenderkumar D, et al. A randomised controlled study of mindfulness meditation versus relaxation therapy in the management of tinnitus. J Laryngol Otol 2017;131:501-7.

31 Kreuzer PM, Goetz M, Holl M, et al. Mindfulness-and bodypsychotherapy-based group treatment of chronic tinnitus: a randomized controlled pilot study. BMC Complement Altern Med 2012;12:235.

32 Roland LT, Lenze EJ, Hardin FM, et al. Effects of mindfulness based stress reduction therapy on subjective bother and neural connectivity in chronic tinnitus. Otolaryngology-Head and Neck Surgery 2015;152:919-26.

33 Gans JJ, O'Sullivan P, Bircheff V. Mindfulness based tinnitus stress reduction pilot study: a symptom Perception-Shift program. Mindfulness 2014;5:322-33.

34 Sadlier M, Stephens SDG, Kennedy V. Tinnitus rehabilitation: a mindfulness meditation cognitive behavioural therapy approach. $J$ Laryngol Otol 2008;122:31-7.

35 Philippot $\mathrm{P}$, Nef F, Clauw L, et al. A randomized controlled trial of Mindfulness-Based cognitive therapy for treating tinnitus. Clin Psychol Psychother 2012;19:411-9.

36 Rademaker MM, Stegeman I, Ho-Kang-You KE, et al. The effect of Mindfulness-Based interventions on tinnitus distress. A systematic review. Front Neurol 2019;10:1135.

37 Gans JJ, Cole MA, Greenberg B. Sustained benefit of MindfulnessBased tinnitus stress reduction (MBTSR) in adults with chronic tinnitus: a pilot study. Mindfulness 2015;6:1232-4.

38 Henry JA, Griest S, Thielman E, et al. Tinnitus functional index: development, validation, outcomes research, and clinical application. Hear Res 2016;334:58-64.

39 Chan A-W, Tetzlaff JM, Altman DG, et al. Spirit 2013 statement: defining standard protocol items for clinical trials. Ann Intern Med 2013;158:200-7.

40 BV C. Castor electronic data capture. Amsterdam, The Netherlands, 2018.

41 Tromp R. De betrouwbaarheid en validiteit van de Nederlandstalige versie van de tinnitus functional index (TFI). Univ Med Cent Groningen, 2014.

42 Trompenaars FJ, Masthoff ED, Van Heck GL, et al. Content validity, construct validity, and reliability of the WHOQOL-Bref in a population of Dutch adult psychiatric outpatients. Qual Life Res 2005;14:151-60.

43 Spinhoven P, Ormel J, Sloekers PP, et al. A validation study of the hospital anxiety and depression scale (HADS) in different groups of Dutch subjects. Psychol Med 1997;27:363-70.

44 Maya Schroevers IN cek en RT. Validatie van de Nederlandstalige versie van de mindful attention awareness scale (MAAS). Gedragstherapie 2008;41:225-40.

45 de Ridder DTD, van Heck GL. Ciss coping inventory for stressful situations. Pearson Clinical \& Talent Assessment 2004.

46 Busner J, Targum SD. The clinical global impressions scale: applying a research tool in clinical practice. Psychiatry 2007;4:28-37.

47 Arrindell WA AA. De Nederlandse symptom Checklist-90-Revised (SCL-90-R): EEN multidimensionale psychopathologie-indicator voor gebruik bij somatische patiënten. Diagnostiek-wijzer 2004;3:105-13. 